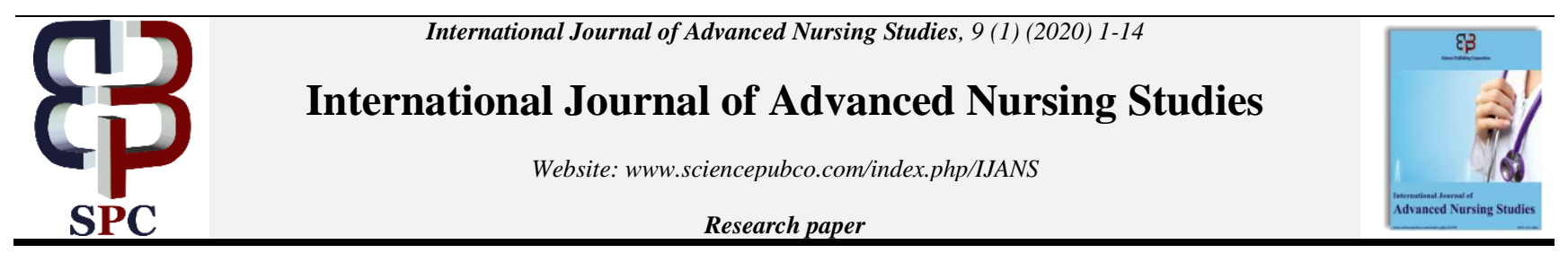

\title{
Perception of women towards contraceptive methods: using health belief model
}

\author{
Noha Mohamed Mahmoud ${ }^{1}$ *, Asmaa Saber Ghaly ${ }^{2}$, Afaf Hassan Ahmed ${ }^{2}$ \\ ${ }^{1}$ Assistance professor of Obstetrics and Gynecologic Nursing, Faculty of Nursing, Alexandria University. \\ ${ }^{2}$ Lecturers of Obstetrics and Gynecologic Nursing, Faculty of Nursing, Alexandria University. Egypt \\ *Corresponding author E-mail: drasmaaghaly@alexu.edu.eg
}

\begin{abstract}
Introduction: The Health Belief Model (HBM) is one of the major conceptual frameworks guiding current research as well as practice in the health sciences. It takes into account the multiple factors present in a person's decisions to live a healthy life, seek help when needed, and maintain periodic check-ups and screening. Aim of the study is to identify perception of women towards contraceptive methods using the health belief model. Research design: A descriptive research design was utilized in this study. Setting: This study was conducted at four family planning clinics affiliated to four maternity hospitals namely: EL- Shatby Maternity University hospital affiliated to Alexandria University, Dar-Ismail Maternity hospital affiliated to Ministry of Health, Faisal hospital affiliated to Health Insurance and Dar ALWelada hospital affiliated to Medical Health Association. Subjects: A convenience sample of 320 women who were seeking family planning services. Tools; Tool I: Contraceptive users' basic data structured interview schedule; Tool II: Contraceptive users' Health Belief scale. Results: Approximately an equal percent $(56.56 \% \& 43.44 \%)$ of the contraceptive users perceived themselves either highly susceptible or moderately susceptible for threat of getting pregnant, respectively. More than three-fourths $(76.25 \%)$ of them had moderate perceived severity to the problems associated with contraceptive use compared to only $23.75 \%$ of them who had high level of perception. As much as $84.37 \%$ of them highly perceived the benefits of contraception uptake. Meanwhile, almost all $(93.44 \%)$ of them moderately perceived barriers associated with contraception utilization. Conclusion: Contraceptive users had high perception related to susceptibility of the threat of pregnancy and benefits of contraceptive methods utilization. Moreover, the vast majority of them had moderate perception to severity and barriers associated with contraception uptake. Last but not least, there was a statistically significant correlation between women's health beliefs and their utilization of contraceptive method.
\end{abstract}

Keywords: Contraceptive Methods; Health Belief Model; Perception of Women.

\section{Introduction}

Unplanned pregnancy (UP) is a global problem that have negative effect on the health of women, their children and families. UP is defined as a pregnancy that is either unwanted or mistimed at the time of conception. Mistimed pregnancy means pregnancy that is desired later in life but not at conception while unwanted pregnancy is that pregnancy that is not wanted then or at any time in the future. The term of unplanned pregnancy is used interchangeably with unintended pregnancy (Ali, Ali, Aziz Ali \& Khuwaja, 2016; Kassa, Berhane \& Worku, 2012). Unplanned pregnancy is considering a crucial health issue in the developing world. Globally, there are 210 million women become pregnant annually, out of whom, 80 million (38\%) of them experience UP and approximately $42-46$ million ( $>50 \%)$ of these UP are terminated and 19 million of them have unsafe abortions. Moreover about 68, 000 women die every year from the complications of these unsafe abortions (Nyarko, 2019). According to Egypt Demographic Health Survey (EDHS) 2014, 16\% of births were not wanted at the conception (El-Zanaty, 2014).

Family planning (FP) plays an integral part in women's life. According to the world health organization (WHO) 2013 FP is defined as the practice that helps individuals or couples to attain the desired number of children through spacing and timing their births. It is achieved through use of contraceptive methods and treatment of infertility. While contraception is any method by which pregnancy is prevented (World Health Organization (WHO), 2013).

Despite the availability of various contraceptive methods for women to choose from, the prevalence of contraceptive methods use remains relatively low especially in developing countries. Low prevalence of contraception use contributes to high rate of unintended pregnancy that indicate that there is still an urgent unmet family planning need and underutilization of modern contraceptive methods (Yanikkerem, Ay \& Piro, 2013). A myriad of different factors associated with women's decision regarding using of family planning methods. These factors are socio-demographic characteristics, effectiveness, availability and accessibility, cost, knowledge and awareness, religious factors, health risks, misconceptions, fear and rumors and women health beliefs, values and attitudes. (Çalıkoğlu et al., 2018)

Women Health beliefs, values and attitudes, are considered as the elements able to shape the development of attitude toward an object (Sensoy et al., 2018). Beliefs are the basic assumptions, ideas, or convections that are accepted as a truth, whether are true or false, within a culture. Attitude refers to the positive or negative feelings or tendencies of an individual about an idea, an object or a symbol. Beliefs and attitudes play an important role in the choice of family planning methods. As family planning behaviors are choices based on these beliefs 
and values; unfortunately, it was found that women don't voluntarily enter health care system as a client until they are suffering from disabling symptoms (Jalang'o et al., 2017). The Health Belief Model (HBM) is one of the major conceptual frameworks guiding current research as well as practice in the health sciences. It takes into account the multiple factors present in a person's decisions to live a healthy life, seek help when needed, and maintain periodic check-ups and screening. The health belief model provides a framework for understanding the potential influence on an individual's decision to make use of available health services (de Peralta, 2011). It states that the perception of a personal health behavior threat is itself influenced by at least three factors: general health values, specific health beliefs about vulnerability to a particular health threat and beliefs about the consequences of the health problem. Once an individual perceives a threat to his/her health and is simultaneously cued to action, and his/her perceived benefits outweighs his/her perceived barriers, then that individual is most likely to undertake the recommended preventive health action. There may be some variables (demographic, socio-psychological, and structural) that can influence an individual's decision (Chernick et al., 2015; Zhao et al., 2012).

The health belief model posits that to be motivated to use contraception, women must consider themselves to be susceptible to the threat of pregnancy (perceived susceptibility), believe that the using contraception may be associated with problems (perceived severity), understand that using contraceptive methods has beneficial effect on women health (perceived benefits).These factors are weighed against the costs of using contraception (perceived barriers). (Chernick et al., 2015; Zhao et al., 2012). The link between women's health beliefs and selection of a contraceptive method remains an area requiring further investigation. So, exploring women's health belief regarding contraceptives using health belief model describes the use of contraceptives among women, understand women's health seeking behavior from an individual level as well as the mechanisms of their decision-making process for contraceptive use and ultimately decrease the risk of an unintended pregnancy and increase contraceptives utilization among women.

\subsection{Aim of the study}

This study aims to identify perception of women towards contraceptive methods using the health belief model.

\subsection{Research question}

What is the perception of women towards contraceptive methods?

\section{Materials and method}

\subsection{Materials}

\subsubsection{Research design}

A descriptive research design was utilized in this study

\subsubsection{Setting}

This study was conducted at four family planning clinics affiliated to four maternity hospitals namely

- EL-Shatby Maternity University Hospital affiliated to Alexandria University.

- Dar-Ismail Maternity Hospital affiliated to Ministry of Health.

- Faisal Hospital affiliated to Health Insurance.

- Dar ALWelada hospital affiliated to Medical Health Association.

The settings were selected because they had an increased turnover of women who are seeking family planning services.

\subsubsection{Subjects}

A convenience sample of 320 women attending the previously mentioned settings were recruited according to the following criteria:

- At reproductive age (18-45 years).

- Female contraceptive user.

The Epi info 7 program was used to estimate the sample size using the Following parameters:

a) Population size $=1800$ over 3 month

b) Expected frequency $50 \%$

c) Acceptable error 5\%

d) Confidence coefficient $95 \%$

e) Minimal sample size 317

- 27 subjects were selected from EL-Shatby Maternity University hospital, 53 subjects were selected from Dar ALWelada hospital, 133 subjects from Dar-Ismail Maternity hospital and 107subjects were selected from Faisal hospital according to turn over of each setting.

\subsubsection{Tools of data collection: Two tools were used for data collection}

Tool I: Contraceptive users' basic data structured interview schedule:

This tool was developed by the researchers after reviewing of literature. It includes four parts:

1) Socio-demographic characteristics such as: the subjects' age, level of education, occupation, marital status, current residence, family type and monthly income.

2) Reproductive history such as: gravidity, parity, number of abortions, stillbirths, number and sex of living children.

3) Medical and surgical history such as hypertension, heart diseases, diabetes, respiratory diseases, anemia, as well as pelvic and abdominal operations.

4) Contraceptive use history such as name of contraceptive method, decision making about using contraceptive method, duration of use, follow up (place and pattern) and side effects.

Tool II: Contraceptive users' Health Belief scale: 
This tool was adapted from two scales Victoria Champion, 1993 (Champion, 1993), and (Becker et al., 1977) to measure health belief among contraceptive users. The tool was translated into Arabic language by the researchers to suit the Egyptian culture. It entails 32 items divided into 4 sections. In general, each item was scored according to a 4-point Likert format from 1 to 4 as follow:

Strongly disagree (1), Disagree (2), Agree (3) and strongly agree (4).

The total score will range from (32-128). Specifically, each item has its own total score and these sections namely:

Firstly, Perceived susceptibility: It included (3items) from 3-12 and it ranked as follow:

- Low (3-5)

- Moderate (6-8)

- $\quad$ High (9-12)

Secondly, perceived severity: It included (7items) from 7-28 and it ranked as follow:

- Low (7-13)

- Moderate (14-21)

- High (22-28)

Thirdly, Perceived benefits: It included (11items) from 11-44 and it ranked as follow:

- Low (11-21)

- Moderate (22-33)

- High (34-44)

Fourthly, Perceived barriers: It included (11items) from 11-44 and it ranked as follow:

- $\quad$ Low (11-21)

- Moderate (22-33)

- $\quad$ High (34-44)

\subsection{METHOD}

The study was executed according to the following steps:

1) An official letter from Faculty of Nursing - University of Alexandria was directed to the responsible authorities of the previously mentioned settings to obtain their permission for conducting the study.

2) Tool (I) was developed by the researchers after review of recent relevant literature.

3) Tool (II) was adapted and translated into Arabic language by the researchers.

4) Tools content validity was tested by a jury of five experts in the field of obstetric and gynaecologic nursing. The recommended modifications were done, and the final form was finalized after proving valid.

5) Tool's reliability (internal consistency) was tested by Cronbach's -Alpha test and the result was reliable $(r=0.87)$ which is statistically accepted.

6) A pilot study was carried out on 32 pregnant women (excluded from the study subjects) from the previously mentioned settings to assure the feasibility of the study, clarity and applicability of the tools, identify obstacles and to calculate the time needed to complete the tool. After pilot study, the tool was revised, reconstructed and made ready for use.

7) Each woman was individually interviewed to collect tool I and tool II after explaining the aim of the study and obtaining family planning services.

8) Each woman was individually interviewed for 20-40 minutes by the researcher. Three days per week (from 8:30 am to 2:00 pm) were specified for data collection over a period of 6 months, started from the beginning of July till the end of December 2018. An average of 10-11 interviews was performed per day.

9) After completion of data collection, the necessary statistical analysis was done.

10) Statistical analysis: The collected data was revised, categorized, coded, computerized, tabulated and analysed using Statistical Package for Social Sciences (SPSS) version 20. The given graphs were constructed using Microsoft excel software version 2013. The following statistical measures were used:

a) Descriptive statistics: included frequency, percent and mean with standard Deviation to describe the scale and categorical data.

b) Analysis of categorical data:

- Monte Carlo MCP test was used to explore relationships between variables.

- $\quad$ P value $\leq 0.05$ was considered statistically significant.

1. Ethical considerations:

For each subject an informed oral consent was obtained after explaining the purpose of the study. In addition, her anonymity, privacy, freedom to withdraw from the study at any time and confidentiality of her data were all emphasized prior to starting the interview.

\section{Results}

Table 1: Number and Percent Distribution of the Study Subjects According to Their Socio-Demographic Characteristics

\begin{tabular}{|c|c|c|}
\hline Socio-demographic characteristics & $\begin{array}{l}\begin{array}{l}\text { No } \\
(\mathrm{n}=320)\end{array} \\
\end{array}$ & $\%$ \\
\hline \multicolumn{3}{|l|}{ Age } \\
\hline - $\quad<25$ & 50 & 15.62 \\
\hline - $\quad 25<35$ & 134 & 41.88 \\
\hline - $\quad \geq 35$ & 136 & 42.50 \\
\hline \multirow{2}{*}{\multicolumn{3}{|c|}{$\begin{array}{l}\text { Mean } \pm \text { SD } \\
\qquad \quad \text { Education }\end{array}$}} \\
\hline & & \\
\hline - Illiterate & 34 & 10.62 \\
\hline - $\quad$ Primary level & 47 & 14.69 \\
\hline - $\quad$ Preparatory level & 21 & 06.56 \\
\hline - Secondary school & 123 & 38.44 \\
\hline - University or higher & 95 & 29.69 \\
\hline \multicolumn{3}{|l|}{ Occupation } \\
\hline - House wife & 165 & 51.56 \\
\hline
\end{tabular}




\begin{tabular}{|c|c|c|}
\hline $\begin{array}{ll}\text { - } & \text { Employee } \\
\text { - } & \text { Worker }\end{array}$ & $\begin{array}{l}75 \\
80\end{array}$ & $\begin{array}{l}23.44 \\
25.00\end{array}$ \\
\hline \multicolumn{3}{|l|}{ Current residence } \\
\hline - Urban & 246 & 76.88 \\
\hline - $\quad$ Rural & 74 & 23.12 \\
\hline \multicolumn{3}{|l|}{ Type of family } \\
\hline - Nuclear & 295 & 92.19 \\
\hline - $\quad$ Extended & 25 & 07.81 \\
\hline $\begin{array}{ll}\text { Perceived income } \\
\text { - } & \text { Adequate } \\
\text { - } & \text { In adequate }\end{array}$ & $\begin{array}{l}266 \\
54\end{array}$ & $\begin{array}{l}83.12 \\
16.88\end{array}$ \\
\hline
\end{tabular}

Table (1) demonstrates the number and percent distribution of the study subjects according to their socio-demographic characteristics. Almost an equal percent of the subjects were either in their late thirties $(42.50 \%)$ or in their late twenties and early thirties (41.88\%). A minority $(15.62 \%)$ of them were in their early twenties. Less than two- fifths $(38.44 \%)$ of them were holding secondary education. Almost one-third $(29.69 \%)$ of them were university graduates. About, one tenth $(10.62 \%)$ of them were illiterate. Slightly more than one- half $(51.56 \%)$ of the study subjects were housewives; while approximately an equal percent $(25 \% \& 23.44 \%)$ of them were either workers or employees, respectively. Nearly, more than three quarters (76.88\%) of the study subjects were urban dwellers. The vast majority (92.19\% $\& 83.12 \%$ ) of them lived in nuclear families and perceived their family income as adequate, respectively.

Table 2: Number and Percent Distribution of the Study Subjects According to Their Family Planning History

\begin{tabular}{|c|c|c|}
\hline Family planning use history & No $(n=320)$ & $\%$ \\
\hline \multicolumn{3}{|l|}{ Previous use of family planning method } \\
\hline - $\quad$ No & 39 & 12.19 \\
\hline - $\quad$ Yes & 281 & 87.81 \\
\hline \multicolumn{3}{|l|}{ Decision making of FP } \\
\hline - The doctor & 155 & 48.44 \\
\hline - The nurse & 19 & 05.94 \\
\hline - The wife herself & 123 & 38.44 \\
\hline - The wife's mother & 23 & 07.18 \\
\hline \multicolumn{3}{|l|}{ Duration of using the method } \\
\hline - $\quad$ From 6 months to 1 year & 38 & 11.87 \\
\hline - From one to two years & 74 & 23.13 \\
\hline - More than two years & 208 & 65.00 \\
\hline \multicolumn{3}{|l|}{ Follow up } \\
\hline - $\quad$ No & 154 & 48.12 \\
\hline - $\quad$ Yes & 166 & 51.88 \\
\hline \multicolumn{3}{|l|}{ Side effects \# } \\
\hline - No & 56 & 17.50 \\
\hline - $\quad$ Yes & 261 & 82.50 \\
\hline - Increase in body weight & 48 & 15.00 \\
\hline - Nausea and vomiting & 5 & 01.56 \\
\hline - Menstrual disorders & 131 & 40.94 \\
\hline - Headache & 48 & 15.00 \\
\hline - Increase the size of the breast & 27 & 08.44 \\
\hline - $\quad$ Spotting of blood from vagina after insertion of IUD & 13 & 04.06 \\
\hline - $\quad$ Back pain and abdominal cramps & 16 & 05.00 \\
\hline - $\quad$ Vaginitis & 13 & 04.06 \\
\hline
\end{tabular}

\# Total is not exclusive.

According to table (2) the majority $(87.81 \%)$ of the subjects were previous users of family planning methods. While slightly more than one-tenth $(12.19 \%)$ of them didn't use any family planning methods before. Regarding decision making for the use of FP methods, it was made by the doctor among almost one-half $(48.44 \%)$ of the subjects. In slightly more than one-third (38.44\%) of them the decision was made by the wives themselves. In almost an equal percent of the subjects it was made either by nurses (5.94\%) or by the mothers of the wives $(7.18 \%)$. Also, two third (65\%) of them used FP method for more than two years. Nearly one- fourth of them (23.13\%)used it for one to two years and a minority $11.87 \%$ of them used it for six months to one year. As much as $51.88 \%$ of them had follow up visits. The majority $(82.50 \%)$ of them had side effect from contraceptive methods which included menstrual disorders among two-fifths (40.94\%) of them, while an equal percent (15\%) of them had either headache or increase in body weight. A minority $8.44 \%$ of them had increase the size of the breast, Also an equal percent $(4.06 \% \& 4.06 \%)$ of them had either spotting of blood from vagina after insertion of IUD or vaginitis.

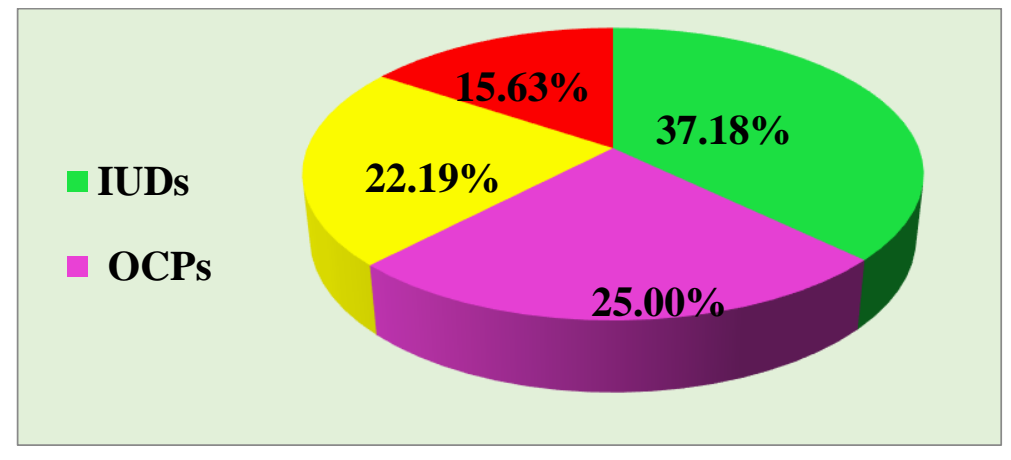


Fig. 1: Percent Distribution of the Study Subjects According to Their Current Contraceptive Methods Utilization.

This figure reflects that more than one-third (37.18\%) of the study subjects were IUDs users. While about one quarter (25\% \& $22.19 \%)$ of them were either OCPs or injectables users, respectively. Only $15.63 \%$ of them was implants users.

Table 3: Number and Percent Distribution of Study Subjects According to Their Perceived Susceptibility and Perceived Severity

\begin{tabular}{|c|c|c|c|c|c|c|c|c|}
\hline \multirow{2}{*}{ Contraceptive user's health beliefs } & \multicolumn{2}{|c|}{ Strongly agree } & \multicolumn{2}{|c|}{ Agree } & \multicolumn{2}{|c|}{ Disagree } & \multicolumn{2}{|c|}{ Strongly disagree } \\
\hline & No & $\%$ & No & $\%$ & No & $\%$ & No & $\%$ \\
\hline \multicolumn{9}{|l|}{ Perceived susceptibility } \\
\hline $\begin{array}{l}\text { - Using contraceptives increase chance of getting } \\
\text { sickness. }\end{array}$ & 13 & 4.06 & 93 & 29.06 & 146 & 45.63 & 68 & 21.25 \\
\hline $\begin{array}{l}\text { - Using contraceptives reduce the chances of un- } \\
\text { intended pregnancy. }\end{array}$ & 143 & 44.69 & 177 & 55.31 & 0.00 & 0.00 & 0.00 & 0.00 \\
\hline $\begin{array}{l}\text { Using contraceptives increase chance of unin- } \\
\text { tended pregnancy. } \\
\text { - Perceived severity }\end{array}$ & 0.00 & 0.00 & 0.00 & 0.00 & 180 & 56.25 & 140 & 43.75 \\
\hline $\begin{array}{l}\text { - Using contraceptives may cause many health } \\
\text { problems. }\end{array}$ & 49 & 15.31 & 127 & 39.69 & 123 & 38.44 & 21 & 6.56 \\
\hline $\begin{array}{l}\text { - Using contraceptives may cause long lasting so- } \\
\text { cial problems. }\end{array}$ & 0.00 & 0.00 & 44 & 13.75 & 213 & 66.56 & 63 & 19.69 \\
\hline $\begin{array}{l}\text { Using contraceptives may cause psychological } \\
\text { problems. }\end{array}$ & 0.00 & 0.00 & 0.00 & 0.00 & 151 & 47.19 & 169 & 52.81 \\
\hline $\begin{array}{l}\text { Not using contraceptives change one's self-es- } \\
\text { teem and self-identity. }\end{array}$ & 42 & 13.13 & 153 & 47.81 & 125 & 39.06 & 0.00 & 0.00 \\
\hline $\begin{array}{l}\text { Not using contraceptives delay one's ambitions } \\
\text { and goals. }\end{array}$ & 57 & 17.81 & 194 & 60.63 & 69 & 21.56 & 0.00 & 0.00 \\
\hline $\begin{array}{l}\text { - Not using contraceptives may cause financial } \\
\text { burden. }\end{array}$ & 202 & 63.13 & 106 & 33.12 & 12 & 3.75 & 0.00 & 0.00 \\
\hline $\begin{array}{l}\text { - Using contraceptives may cause sexual prob- } \\
\text { lems. }\end{array}$ & 14 & 4.38 & 108 & 33.74 & 190 & 59.38 & 8 & 2.50 \\
\hline
\end{tabular}

Table (3) delineates distribution of study subjects according to their perceived susceptibility. Nearly, one- half (45.63\%) of the study subjects disagreed that using contraceptives increase chance of getting sickness compared to one-third (29.06\%) of them who agreed with it. About one-half $(44.69 \%$ \& $55.31 \%)$ of the study subjects were either strongly agreed or agreed that using contraceptives reduce the chances of unintended pregnancy respectively. While as more than one-half $(56.25 \%)$ of the study subjects disagreed that using contraceptives increase chance of unintended pregnancy.

Considering perceived severity regarding contraception utilization, more than one-third $(39.69 \%)$ of the study subjects agreed that using contraceptives may cause many health problems. As much as $66.56 \%$ of the study subjects disagreed that using contraceptives may cause long lasting social problems compared to only $13.75 \%$ of them who were agreed. Approximately one- half $(52.81 \% \& 47.19 \%)$ of the study subjects were either strongly disagreed or disagreed that using contraceptives may cause psychological problems respectively. Slightly less than one- half $(47.81 \%)$ of the study subjects agreed that not using contraceptives change one's self-esteem and self-identity compared to about two-fifths $(39.06 \%)$ of them who were disagreed. However about two-thirds $(60.63 \%)$ of them agreed that not using contraceptives delay one's ambitions and goals. Also, two- thirds (63.13\%) of the study subjects strongly agreed that not using contraceptives may cause financial burden. As much as $59.38 \%$ of them disagreed that using contraceptives may cause sexual problems.

Table 4: Number and Percent Distribution of Study Subjects According to Their Perceived Benefits

\begin{tabular}{|c|c|c|c|c|c|c|c|c|}
\hline \multirow{2}{*}{ Contraceptive user's health beliefs } & \multicolumn{2}{|c|}{ Strongly agree } & \multicolumn{2}{|c|}{ Agree } & \multicolumn{2}{|c|}{ Disagree } & \multicolumn{2}{|c|}{ Strongly disagree } \\
\hline & No & $\%$ & No & $\%$ & No & $\%$ & No & $\%$ \\
\hline \multicolumn{9}{|l|}{ Perceived benefits } \\
\hline $\begin{array}{l}\text { - Using contraceptives reduce the risk of unintended preg- } \\
\text { nancy. }\end{array}$ & 175 & 54.68 & 139 & 43.44 & 6 & 1.88 & 0.00 & 0.00 \\
\hline - Using contraceptives prevent many diseases. & 56 & 17.50 & 164 & 51.25 & 100 & 31.25 & 0.00 & 0.00 \\
\hline - Using enhance one's self image and self-identity. & 67 & 20.94 & 250 & 78.12 & 3 & 0.94 & 0.00 & 0.00 \\
\hline - Using contraceptives regulate menstrual cycle. & 15 & 4.68 & 126 & 39.38 & 179 & 55.94 & 0.00 & 0.00 \\
\hline - Using contraceptives organize family size. & 237 & 74.06 & 83 & 25.94 & 0.00 & 0.00 & 0.00 & 0.00 \\
\hline $\begin{array}{l}\text { - Using contraceptives help to arrange one's duties and re- } \\
\text { sponsibilities. }\end{array}$ & 252 & 78.75 & 56 & 17.50 & 12 & 3.75 & 0.00 & 0.00 \\
\hline - Using contraceptives are easy. & 167 & 52.19 & 136 & 42.50 & 17 & 5.31 & 0.00 & 0.00 \\
\hline - Using contraceptives are cost effective. & 224 & 70.00 & 96 & 30.00 & 0.00 & 0.00 & 0.00 & 0.00 \\
\hline - Using contraceptives are reversible & 167 & 52.19 & 136 & 42.50 & 17 & 5.31 & 0.00 & 0.00 \\
\hline - Using contraceptives are safe and long lasting. & 132 & 41.25 & 131 & 40.94 & 57 & 17.81 & 0.00 & 0.00 \\
\hline $\begin{array}{l}\text { - Using contraceptives positively affect intimate relation- } \\
\text { ship. }\end{array}$ & 90 & 28.13 & 221 & 69.06 & 9 & 2.81 & 0.00 & 0.00 \\
\hline
\end{tabular}

Table (4) reflects that approximately one- half $(54.68 \% \& 43.33 \%)$ of the study subjects were either strongly agreed or agreed that using contraceptives reduce the risk of unintended pregnancy. About one- half $(51.25 \%)$ of them were agreed that using contraceptives prevent many diseases compared to only $31.25 \%$ of them who were disagreed. Slightly more than three quarters $(78.12 \%)$ of the study subjects agreed that using contraceptives enhance one's self image and self-identity. As much as $55.94 \%$ of them disagreed that using contraceptives regulate menstrual cycle. Approximately three -fourths $(74.06 \%$ \& $78.75 \%$ \& $70 \%)$ of them were strongly agreed that using contraceptives organize family size, help to arrange one's duties and responsibilities and cost effective, respectively. An equal percent (52.19\%) of the study subjects strongly agreed that using contraceptives are easy to use and reversible. About two-fifths (41.25\%) of the study subjects strongly agreed that using contraceptives are safe and long lasting. As much as $69.06 \%$ of them agreed that using contraceptive and positively affect intimate relationship. 
Table 5: Number and Percent Distribution of Study Subjects According to Their Perceived Barriers

\begin{tabular}{|c|c|c|c|c|c|c|c|c|}
\hline \multirow{2}{*}{ Contraceptive user's health beliefs } & \multicolumn{2}{|c|}{ Strongly agree } & \multicolumn{2}{|c|}{ Agree } & \multicolumn{2}{|c|}{ Disagree } & \multicolumn{2}{|c|}{ Strongly disagree } \\
\hline & No & $\%$ & No & $\%$ & No & $\%$ & No & $\%$ \\
\hline \multicolumn{9}{|l|}{ Perceived barriers } \\
\hline $\begin{array}{l}\text { - Using contraceptives through male doctors are embar- } \\
\text { rassing issue. }\end{array}$ & 42 & 13.13 & 190 & 59.38 & 86 & 26.86 & 2 & 0.63 \\
\hline - Using contraceptives are time consuming. & 0.00 & 0.00 & 84 & 26.25 & 207 & 64.69 & 29 & 9.06 \\
\hline $\begin{array}{l}\text { Using contraceptives are negatively affected by long dis- } \\
\text { tance and waiting time. }\end{array}$ & 7 & 2.19 & 71 & 22.19 & 217 & 67.81 & 25 & 7.81 \\
\hline $\begin{array}{l}\text { - Using contraceptives negatively affects the intimate rela- } \\
\text { tionship. }\end{array}$ & 0.00 & 0.00 & 0.00 & 0.00 & 226 & 70.63 & 94 & 29.37 \\
\hline - Using contraceptives cause infertility. & 0.00 & 0.00 & 72 & 22.50 & 141 & 44.06 & 107 & 33.44 \\
\hline $\begin{array}{l}\text { - Using contraceptives associated with developing can- } \\
\text { cers. }\end{array}$ & 18 & 5.62 & 91 & 28.44 & 174 & 54.38 & 37 & 11.56 \\
\hline $\begin{array}{l}\text { Using contraceptives doesn't protect against sexually } \\
\text { transmitted diseases. }\end{array}$ & 14 & 4.38 & 187 & 58.44 & 102 & 31.88 & 17 & 5.31 \\
\hline $\begin{array}{l}\text { - Using contraceptives may cause abdominal cramps or } \\
\text { back ache. }\end{array}$ & 41 & 12.81 & 137 & 42.81 & 110 & 34.38 & 32 & 10.00 \\
\hline - Using contraceptives may cause menstrual irregularities. & 59 & 18.44 & 131 & 40.94 & 121 & 37.81 & 9 & 2.81 \\
\hline - Using contraceptives require the initiation of new habits. & 4 & 1.25 & 158 & 49.38 & 136 & 42.50 & 22 & 6.87 \\
\hline - Using contraceptives are sophisticated and need skills & 0.00 & 0.00 & 72 & 22.50 & 141 & 44.06 & 107 & 33.44 \\
\hline
\end{tabular}

Table (5) illustrates that more than one- half $(59.38 \%)$ of the study subjects agreed that using contraceptives through male doctors are an embarrassing issue. More than two-thirds $(64.69 \%$ \& $67.81 \%$ \& $70.63 \%)$ of them disagreed that using contraceptives are time consuming, negatively affected by long distance and waiting time and negatively affect intimate relationship, respectively. An equal percent (44.06\%) of the study subjects disagreed that using contraceptives can either cause infertility or sophisticated and need skills. However more than one-half of them $(54.38 \%)$ of them disagreed that using contraceptives associated with developing cancers compared to only $11.56 \%$ of them who were strongly disagreed. More than one-half $(58.44 \%)$ of the study subjects agreed that using contraceptives doesn't protect against sexually transmitted diseases. More than two-fifths $(42.81 \%$ \& 40.49\% \& 49.38\%) of the study subjects agreed that using contraceptives may cause abdominal cramps or backache, may cause menstrual irregularities as well as require initiation of new habits, respectively.

Table 6: Distribution of the Study Subjects According to Their Overall Contraceptive Health Beliefs

\begin{tabular}{|c|c|c|}
\hline $\begin{array}{l}\text { Contraceptive user's health beliefs } \\
\text { Perceived susceptibility }\end{array}$ & No $(n=320)$ & $\%$ \\
\hline - $\quad$ Moderate & 139 & 43.44 \\
\hline - High & 181 & 56.56 \\
\hline \multicolumn{3}{|l|}{ Perceived severity } \\
\hline - $\quad$ Moderate & 244 & 76.25 \\
\hline - High & 76 & 23.75 \\
\hline \multicolumn{3}{|l|}{ Perceived benefits } \\
\hline - Moderate & 50 & 15.63 \\
\hline - High & 270 & 84.37 \\
\hline \multicolumn{3}{|l|}{ Perceived barriers } \\
\hline - Low & 21 & 06.56 \\
\hline - $\quad$ Moderate & 299 & 93.44 \\
\hline
\end{tabular}

The table reveals that approximately an equal percent $(56.56 \% \& 43.44 \%)$ of the contraceptive users perceived themselves either highly susceptible or moderately susceptible for threat of getting pregnant, respectively. More than three-fourths (76.25\%) of them had moderate perceived severity to the problems associated with contraceptive use compared to only $23.75 \%$ of them who had high level of perception. As much as $84.37 \%$ of them highly perceived the benefits of contraception uptake. Meanwhile, almost all (93.44\%) of them moderately perceived barriers associated with contraception utilization.

Table 7: Relationship between Subjects' Perceived Susceptibility, Perceived Severity and Their Utilization of Contraceptive Methods

\begin{tabular}{|c|c|c|c|c|c|c|c|c|c|c|}
\hline \multirow{3}{*}{ Utilization of contraceptive methods } & \multicolumn{5}{|c|}{ Perceived susceptibility } & \multicolumn{5}{|c|}{ Perceived severity } \\
\hline & \multicolumn{2}{|c|}{ Moderate } & \multicolumn{2}{|c|}{ High } & \multirow[t]{2}{*}{ Significance $\left({ }^{\mathrm{MC}} \mathrm{P}\right)$} & \multicolumn{2}{|c|}{ Moderate } & \multicolumn{2}{|c|}{ High } & \multirow{2}{*}{ Significance $\left({ }^{\mathrm{MC}} \mathrm{P}\right)$} \\
\hline & No & $\%$ & No & $\%$ & & No & $\%$ & No & $\%$ & \\
\hline - $\quad$ IUD $(\mathrm{N}=119)$ & 40 & 33.61 & 79 & 66.39 & \multirow{4}{*}{$0.000 *$} & 89 & 74.79 & 30 & 25.21 & \multirow{4}{*}{$0.000 *$} \\
\hline - $\quad$ OCPs $(\mathrm{N}=80)$ & 45 & 56.25 & 35 & 43.75 & & 58 & 72.50 & 22 & 27.50 & \\
\hline - Injectables $(\mathrm{N}=71)$ & 47 & 66.20 & 24 & 33.80 & & 67 & 94.37 & 4 & 05.63 & \\
\hline Implants $(\mathrm{N}=50)$ & 7 & 14.00 & 43 & 86.00 & & 30 & 60.00 & 20 & 40.00 & \\
\hline
\end{tabular}

${ }^{\mathrm{MC}} \mathrm{P}: \mathrm{P}$ value based on Monte Carlo Exact Probability Test

* Significant at $\mathrm{P}<0.05$.

Table (7) denotes that there was a statistically significant correlation between study subjects' perceived susceptibility and their utilization of contraceptive methods ( $\mathrm{MCP}=0.00)$. Where a substantial proportion $(66.39 \% \& 86.00 \%)$ of IUDs and implants users perceived themselves highly susceptible for threat of getting pregnant compared to only (43.75\% \& 33.80\%) of OCPs and injectables users, respectively. Also, the table exhibits that there was a statistically significant correlation between study subject's perceived severity and their utilization of contraceptives $(\mathrm{MCP}=0.00)$. Specifically, Two- fifths $(40 \%)$ of the study subjects who were implants users had high perceived severity to problems associated with its utilization compared to approximately an equal percent $(25.21 \% \& 27.50 \%)$ of those who were either IUDs or OCPs users, respectively. 
Table 8: Relationship between Study Subjects' Perceived Benefits, Perceived Barriers and Their Utilization of Contraceptive Methods

\begin{tabular}{|c|c|c|c|c|c|c|c|c|c|c|}
\hline \multirow{3}{*}{ Utilization of contraceptive methods } & \multicolumn{4}{|c|}{ Perceived benefits } & \multirow{3}{*}{ Significance $\left({ }^{\mathrm{MC}} \mathrm{P}\right)$} & \multicolumn{4}{|c|}{ Perceived barriers } & \multirow{3}{*}{ Significance $\left({ }^{\mathrm{MC}} \mathrm{P}\right)$} \\
\hline & \multicolumn{2}{|c|}{ s Moderate } & \multicolumn{2}{|l|}{ High } & & \multicolumn{2}{|l|}{ Low } & \multicolumn{2}{|c|}{ Moderate } & \\
\hline & No & $\%$ & No & $\%$ & & No & $\%$ & No & $\%$ & \\
\hline - $\quad$ IUD $(\mathrm{N}=119)$ & 6 & 5.04 & 113 & 94.96 & & 119 & 100.00 & 0 & 00.0 & \\
\hline - $\quad$ OCPs $(\mathrm{N}=80)$ & 20 & 25.00 & 60 & 75.00 & & 11 & 13.75 & 69 & 86.25 & \\
\hline - Injectables $(\mathrm{N}=71)$ & 20 & 28.17 & 51 & 71.83 & 0 & 6 & 08.45 & 65 & 91.55 & $0.002 *$ \\
\hline - Implants $(\mathrm{N}=50)$ & 4 & 08.00 & 46 & 92.00 & & 4 & 08.00 & 46 & 92.00 & \\
\hline
\end{tabular}

${ }^{\mathrm{MC}} \mathrm{P}: \mathrm{P}$ value based on Monte Carlo Exact Probability Test.

* Significant at $\mathrm{P}<0.05$.

Table (8) illustrates that there was a statistically significant positive correlation between study subject's perceived benefits and their contraceptive methods utilization ( $\mathrm{MCP}=0.00)$. Almost all $(94.96 \% \& 92 \%)$ of the subjects who were either IUDs or implants users had high perceived benefits to their contraception uptake compared to three-fourths ( $75 \% \& 71.83 \%$ ) of those who were either OCPs or injectables users, respectively.

The table also, delineates that there was a statistically significant correlation between study subject's perceived barriers and their contraceptive methods utilization $(\mathrm{MCP}=0.02)$ where the vast majority $(86.25 \% \& 91.55 \% \& 92 \%)$ of the study subjects who were OCPs or injectables or implants users had moderate perceptions to the barriers associated with contraception utilization compared to none of IUDs users.

Table 9: The Relationship between Subjects' Perceived Susceptibility and Perceived Severity and Their Socio-Demographic Characteristics

\begin{tabular}{|c|c|c|c|c|c|c|c|c|c|c|}
\hline \multirow{3}{*}{ socio-demographic characteristics } & \multirow{2}{*}{\multicolumn{4}{|c|}{$\begin{array}{l}\text { Perceived susceptibility } \\
\text { Moderate }(\mathrm{N}=139) \text { High }(\mathrm{N}=181)\end{array}$}} & \multirow{3}{*}{ Significance $\left({ }^{\mathrm{MC}} \mathrm{P}\right)$} & \multicolumn{5}{|c|}{ Perceived severity } \\
\hline & & & & & & Mod & $(\mathrm{N}=2$ & $\mathrm{Hig}$ & $\mathrm{v}=76)$ & \multirow{2}{*}{ Significance $\left({ }^{\mathrm{MC}} \mathrm{P}\right)$} \\
\hline & No & $\%$ & No & $\%$ & & No & $\%$ & No & $\%$ & \\
\hline \multicolumn{11}{|l|}{ Age (years) } \\
\hline$\bullet<25$ & 18 & 12.95 & 32 & 17.68 & \multirow{3}{*}{0.077} & 39 & 15.98 & 11 & 14.47 & \multirow{3}{*}{0.078} \\
\hline - $25<35$ & 68 & 48.92 & 66 & 36.46 & & 94 & 38.53 & 40 & 52.64 & \\
\hline - $\quad->35$ & 53 & 38.13 & 83 & 45.86 & & 111 & 45.49 & 25 & 32.89 & \\
\hline \multicolumn{11}{|l|}{ Level of education } \\
\hline - Illiterate & 16 & 11.51 & 18 & 09.94 & \multirow{5}{*}{$0.000^{*}$} & 27 & 11.07 & 7 & 09.21 & \multirow{5}{*}{$0.040^{*}$} \\
\hline - Primary level & 33 & 23.74 & 14 & 07.74 & & 43 & 17.62 & 4 & 05.26 & \\
\hline - Preparatory level & 12 & 08.63 & 9 & 04.98 & & 18 & 07.38 & 3 & 03.95 & \\
\hline - Secondary level & 53 & 38.13 & 70 & 38.67 & & 87 & 35.66 & 36 & 47.37 & \\
\hline - University or higher & 25 & 17.99 & 70 & 38.67 & & 69 & 28.27 & 26 & 34.21 & \\
\hline \multicolumn{11}{|l|}{ Occupation } \\
\hline - Housewife & 69 & 49.64 & 96 & 53.04 & \multirow{3}{*}{$0.000^{*}$} & 124 & 50.82 & 41 & 53.95 & \multirow{3}{*}{0.071} \\
\hline - Worker & 49 & 35.25 & 26 & 14.36 & & 64 & 26.23 & 11 & 14.47 & \\
\hline - Employee & 21 & 15.11 & 59 & 32.60 & & 56 & 22.95 & 24 & 31.58 & \\
\hline \multicolumn{11}{|l|}{ Current residence } \\
\hline - Urban & 106 & 76.26 & 140 & 77.35 & \multirow{2}{*}{0.819} & 189 & 77.46 & 57 & 75.00 & \multirow{2}{*}{0.657} \\
\hline - Rural & 33 & 23.74 & 41 & 22.65 & & 55 & 22.54 & 19 & 25.00 & \\
\hline \multicolumn{11}{|l|}{ Perceived income } \\
\hline - Adequate & 101 & 72.66 & 165 & 91.16 & \multirow[t]{2}{*}{$0.000^{*}$} & 208 & 85.25 & 58 & 76.32 & \multirow[t]{2}{*}{0.070} \\
\hline - In adequate & 38 & 27.34 & 16 & 08.84 & & 36 & 14.75 & 18 & 23.68 & \\
\hline \multicolumn{11}{|l|}{ Family type } \\
\hline - Nuclear & 139 & 100.0 & 156 & 86.19 & $0.000^{*}$ & 231 & 94.67 & 64 & 84.21 & $0.003^{*}$ \\
\hline - Extended & 0 & 00.00 & 25 & 13.81 & & 13 & 05.33 & 12 & 15.79 & \\
\hline
\end{tabular}

${ }^{\mathrm{MC}} \mathrm{P}: \mathrm{P}$ value based on Monte Carlo Exact Probability Test.

* Significant at $\mathrm{P}<0.05$.

Table (9) reflects that there was a statistically significant correlation between study subjects' perceived susceptibility and some of their socio-demographic characteristics namely, education $(\mathrm{MCP}=00.00)$, occupation $(\mathrm{MCP}=00.00)$, perceived income $(\mathrm{MCP}=00.00)$ and type of family $(\mathrm{MCP}=0.002)$.Specifically high perceived susceptibility to threat of getting pregnant was evident among an equal percent (38.67\%) of the subjects who were either university graduates or secondary level compared to only $9.94 \%$ \& $7.74 \%$ of those who were either illiterate or were holding primary certificates, respectively. Slightly more than one-half $(53.04 \%)$ of the study subjects who were housewives perceived themselves highly susceptible to the threat of getting pregnant compared to only $14.36 \%$ \& $32.60 \%$ of those who were either workers or employee, respectively. The vast majority (91.16\%) of those with high perceived susceptibility had adequate income compared to only $8.84 \%$ of them who had inadequate income. Meanwhile, all (100\%) the subjects with moderate perceived susceptibility to pregnancy had nuclear families.

Moreover, a statistically significant correlation was noticed between subjects' perceived severity to the problems associated with contraception use and level of education $(\mathrm{MCP}=0.040)$ and type of family $(\mathrm{MCP}=0.003)$. Whereas a substantial portion $(47.37 \% \& 34.21 \%)$ of those with high perceived severity to problems associated contraception uptake were holding either secondary certificates or university graduates, respectively compared to only $3.95 \%$ of them who were holding preparatory certificates. Moderate perceived severity was evident among almost all $(94.67 \%)$ of the study subjects with nuclear families compared to only $5.33 \%$ of them.

Table 10: The Relationship between Subjects' Perceived Benefits and Perceived Barriers and Their Socio-Demographic Characteristics

\begin{tabular}{|c|c|c|c|c|c|c|c|c|c|c|}
\hline \multirow{3}{*}{ socio-demographic characteristics } & \multicolumn{5}{|c|}{ Perceived benefits } & \multicolumn{5}{|c|}{ Perceived barriers } \\
\hline & \multicolumn{4}{|c|}{ Moderate $(\mathrm{N}=50)$ High $(\mathrm{N}=270)$} & \multirow[t]{2}{*}{$\begin{array}{l}\text { Significance } \\
\text { (MCP) }\end{array}$} & \multicolumn{2}{|c|}{ Low $(\mathrm{N}=21)$} & \multicolumn{2}{|c|}{$\begin{array}{l}\text { Moderate } \\
(\mathrm{N}=299)\end{array}$} & \multirow{2}{*}{ Significance $\left({ }^{\mathrm{MC}} \mathrm{P}\right)$} \\
\hline & No & $\%$ & No & $\%$ & & No & $\%$ & No & $\%$ & \\
\hline \multicolumn{11}{|l|}{ Age (years) } \\
\hline$-<25$ & 1 & 02.00 & 49 & 18.15 & & 3 & 14.29 & 47 & 15.72 & \\
\hline - $25<35$ & 38 & 76.00 & 96 & 35.55 & $0.000^{*}$ & 14 & 66.66 & 120 & 40.13 & $0.038 *$ \\
\hline - $\quad->35$ & 11 & 22.00 & 125 & 46.30 & & 4 & 19.05 & 132 & 44.15 & \\
\hline
\end{tabular}




\begin{tabular}{|c|c|c|c|c|c|c|c|c|c|c|}
\hline \multicolumn{11}{|l|}{ Level of education } \\
\hline - Illiterate & 2 & 04.00 & 32 & 11.85 & \multirow{5}{*}{$0.000^{*}$} & 0 & 00.00 & 34 & 11.37 & \multirow{5}{*}{0.147} \\
\hline - Primary level & 1 & 2.00 & 46 & 17.04 & & 2 & 09.52 & 45 & 15.05 & \\
\hline - Preparatory level & 8 & 16.00 & 13 & 04.82 & & 2 & 09.52 & 19 & 06.35 & \\
\hline - Secondary level & 30 & 60.00 & 93 & 34.44 & & 12 & 57.14 & 111 & 37.12 & \\
\hline - University or higher & 9 & 18.00 & 86 & 31.85 & & 5 & 23.82 & 90 & 30.11 & \\
\hline \multicolumn{11}{|l|}{ Occupation } \\
\hline - Housewife & 30 & 60.00 & 135 & 50.00 & \multirow{3}{*}{0.072} & 18 & 85.72 & 147 & 49.16 & \multirow{3}{*}{$0.005 *$} \\
\hline - Worker & 14 & 28.00 & 61 & 22.59 & & 2 & 09.52 & 73 & 24.42 & \\
\hline - Employee & 6 & 12.00 & 74 & 27.41 & & 1 & 04.76 & 79 & 26.42 & \\
\hline \multicolumn{11}{|l|}{ Current residence } \\
\hline - Urban & 47 & 94.00 & 199 & 73.70 & \multirow[t]{2}{*}{$0.002 *$} & 17 & 80.95 & 229 & 76.59 & \multirow[t]{2}{*}{0.647} \\
\hline - Rural & 3 & 06.00 & 71 & 26.30 & & 4 & 19.05 & 70 & 23.41 & \\
\hline \multicolumn{11}{|l|}{ Perceived income } \\
\hline - Adequate & 35 & 70.00 & 231 & 85.56 & \multirow[t]{2}{*}{0.007} & 21 & 100.00 & 245 & 81.94 & \multirow[t]{2}{*}{$0.033 *$} \\
\hline - In adequate & 15 & 30.00 & 39 & 14.44 & & 0 & 00.00 & 54 & 18.06 & \\
\hline \multicolumn{11}{|l|}{ Family type } \\
\hline - Nuclear & 50 & 100.00 & 245 & 90.74 & \multirow[t]{2}{*}{$0.025^{*}$} & 21 & 100.00 & 274 & 91.64 & \multirow[t]{2}{*}{0.168} \\
\hline - Extended & 0 & 00.00 & 25 & 09.26 & & 0 & 00.00 & 25 & 08.36 & \\
\hline
\end{tabular}

${ }^{\mathrm{MC}} \mathrm{P}: \mathrm{P}$ value based on Monte Carlo Exact Probability Test.

* Significant at $\mathrm{P}<0.05$.

Table (10) demonstrates that there was a statistically significant correlation between study subjects perceived benefits of contraceptive methods utilization and their age $(\mathrm{MCP}=0.00)$, level of education $(\mathrm{MCP}=0.00)$, and place of residence $(\mathrm{MCP}=0.002) . \mathrm{Specifically}$, moderate perceived benefits of contraception utilization was obvious among three-fourths $(76 \%)$ of the study subjects who were in their late twenties and early thirties, respectively, compared to only $2 \%$ \& $22 \%$ of those who were either in their early twenties or late thirties, respectively. Almost an equal percent $(34.44 \% \& 31.85 \%)$ of the subjects who were holding either secondary certificates or were university graduates had high perceived benefits compared to only $11.85 \%$ \& $17.04 \%$ of those who were either illiterate or were holding primary certificates, respectively. Nearly three quarters $(73.70 \%)$ of urban indwellers had high perceived benefits compared to one quarter (26.30\%) of rural ones. Moreover, high perceived benefits were observed among the majority $(90.74 \%)$ of the subjects with nuclear families compared to only one-tenth $(9.26 \%)$ of those who had extended families.

On the contrary, a statistically significant correlation was noticed between study subjects' perceived barriers and their age $(\mathrm{MCP}=0.038)$, occupation $(\mathrm{MCP}=0.005)$ and their perceived income $(\mathrm{MCP}=0.033)$. Specifically, low perceived barriers of contraception utilization were evident among two-thirds $(66.66 \%)$ of the study subjects who were in their late twenties or early thirties compared to only approximately an equal percent $(14.29 \% \& 19.05 \%)$ of the subjects who were either in their early twenties or late thirties, respectively. Moderate perceived barriers to the problem associated with contraception use were present among almost one-half $(49.16 \%)$ of the study subjects who were housewives compared to only $(24.42 \%$ \& $26.42 \%)$ who were either worker or employee, respectively. All (100\%) of the study subjects with low perceived barriers had adequate income.

\section{Discussion}

Contraception use still remaining as paramount importance to women's health. The United Nation 2015 reported that $64 \%$ of married women used contraceptive methods globally. However, contraceptive use was much lower $40 \%$ in the developing countries .Use of a contraceptive methods help couples plan their family by avoiding undesired pregnancies and consequently serving their intention to stop or postpone childbearing (Hettiarachchi \& Gunawardena, 2012; United Nations, 2015).

Family planning services are a necessity in health systems throughout the world. They are one of the most cost-effective ways to reduce many crucial problems. It has positive impact on the mother, child, and potentially society. Today, Many FP programs focus on providing good quality of reproductive health care. Good quality care includes provision of a range of contraceptive methods, sensitive counselling, and meeting reproductive health needs. As quality improves, women become more satisfied, better able to perform informed choices use contraception effectively and continue using them(Tessema, Streak Gomersall, Mahmood \& Laurence, 2016).

It has been estimated that meeting women's need for modern contraceptives would prevent about one quarter to one-third of all maternal deaths, saving 140,000 to150, 000 lives a year. Women's health beliefs are one of the factors that affect the women's decision regarding contraception use. Health beliefs explain the attitudes, perceptions and values of women about contraception use (Sensoy et al., 2018).So ,this study aims to identify health beliefs among female contraceptive users.

Utilization of contraceptive methods:

The present study revealed that more than one-third (37.18\%) of the study subjects were using IUDs. This means that most women would prefer IUDs rather than others family planning methods. According to Egypt demographic health survey (EDHS)2014, IUD is the most widely used family method among $30 \%$ of the Egyptian women (El-Zanaty, 2014). This could be attributed to the fact about IUDs advantages such as IUD is cost- effective, safe, long acting, doesn't affect lactation and also, the fertility returns soon after its removal (Sanders et al., 2017).

This finding is congruent with four researches, (Gharaibeh, Oweis, Shakhatreh \& Froelicher, 2011) who studied factors associated with contraceptive use among Jordanian Muslim women: implications for health and social policy in Jordan. They revealed that IUD was the most preferred method among 44\% of Jordanian women. Also, (Kahraman et al., 2012) who studied factors influencing contraception method choices in Turkey. They reported that IUD was used by $46.40 \%$ of Turkish women. As well as, (Hamdy, 2014) who conducted a study to assess pattern of contraception utilization among women attending to the well-baby clinics in Egypt. She revealed that IUDs were utilized by $47 \%$ of Egyptian women. Finally,(Clifford, Johnscy, Pratidnya, Rachel \& Reshma, 2015) who conducted a descriptive study to assess the perception and practice of selected contraceptive methods among target population in selected areas of Mumbai. They illustrated that $42.85 \%$ of Indian women were IUDs users.

This finding is in disagreement with four researches. Firstly, (Huda et al., 2017) who studied contraceptive practices among married women of reproductive age in Bangladesh (Huda et al., 2017).They reported that IUD was used by only.6\% of Bangladeshi women. Secondly, (Asadisarvestani, Khoo, Malek, Yasin \& Ahmadi, 2017) who conducted a study titled determinants of contraceptive usage among married women in Shiraz, Iran. They revealed that IUD was used by only $6.6 \%$ of Iranian women. Thirdly, (Bamufleh, Al-Zahrani \& Yousuf, 2017) 
whose study titled contraceptive knowledge and use in Saudi Arabia. They reported that IUDs were used by only $22 \%$ of Saudi women. Fourthly,(Ukegbu, Onyeonoro, Nwokeukwu \& Okafor, 2018) who studied contraceptive method preferences, use and satisfaction among women of reproductive age in Nigeria. They illustrated that IUD was the least common method used by only $2.30 \%$ of Nigerian women. The finding of present study revealed that one quarter (25\%) of women were OCPs users it means that OCPs was the second utilized method preferred by study subjects. According to EDHS 2014, about 16\% of Egyptian women are OCPs users (El-Zanaty, 2014). This could be attributed to their cost effectiveness, availability, reversibility, doesn't require interruption before sexual intercourse and doesn't require self-manipulation.

This result is in line with the findings of (Megabiaw, 2012) who done a study titled awareness and utilization of modern contraception among street women in North-West Ethiopia. They revealed 30\% of Ethiopian women were OCPs users. In addition to (Envuladu et al., 2012) who studied utilization of modern contraceptives among female traders in Nigeria. Their results illustrated that oral contraceptive pills were utilized by $18.2 \%$ of Nigerian women. Also, (Samim, 2014) who conducted a study about prevalence of family planning methods among married women aged 15-49 years in one rural area in Afghanistan. They revealed that 31\% of Afghani women were OPCs users. Finally, (Eliason, Bockarie \& Eliason, 2018) who conducted a study titled postpartum fertility behaviours and contraceptive use among women in Ghana. They revealed that $20.6 \%$ of Ghanaian women were using OCPs.

Yet, this finding is inconsistent with two researches. First: the previously mentioned one (Kahraman et al., 2012).They reported that only $5.2 \%$ of Turkish women used oral contraceptive pills. Second: (Alemayehu et al., 2018) whose study titled prevalence and determinants of contraceptive utilization among married women in northwest Ethiopia. They reported that only $8.4 \%$ of Ethiopian women were OCPs users. The contradiction between the present study result and the findings of the other studies may be attributed to variation in sample size where sample size in the present study is only 320 but it was 4022 in the Turkish study and 8271in the Ethiopian one.

The finding of the present study illustrated that less than one quarter $(22.19 \%)$ of the study subjects were injectables users. This could be attributed to the fact that many women preferred injectables due to their cost effectiveness, availability, reversibility as fertility returns within 2 to 3 months after stopping the method (United Nations, 2015).

This finding is consistent with (El Shamy, Abdelsatar \& Awad, 2013) who had studied the contraceptive knowledge and use among women attending primary health care centres of Al Dawahe District in Port Said Governorate. They reported that $30 \%$ of women were injectables users. Also, the previously mentioned study conducted by (Samim, 2014). They reported that 30.2\% of Afghani women were injectables users. At last,(Ilori, Ilori \& Awodutire, 2017) who conducted a study about contraceptive practices among married women of reproductive age group in Nigeria. They revealed that $27.2 \%$ of Nigerian women were injectables users.

However, this same result is contradicted with the findings of four studies. First :(Onokerhoraye \& Dudu, 2016) who conducted a study titled knowledge and practice of family planning by women of childbearing age in Nigeria. They revealed that $58.9 \%$ of rural Nigerian women and $69.5 \%$ of urban Nigerian women were injectables users. Second: (Endriyas et al., 2017) who had studied contraceptive utilization and associated factors among women of reproductive age group in Ethiopia. They also revealed that injectable contraceptive method was the most common method used by $(69.10 \%)$ of the Ethiopian women. Third: the previously mentioned one(Alemayehu et al., 2018). They reported that $81.3 \%$ of Ethiopian women were injectables users. Fourth:(Ontiri et al., 2019) who conducted a study titled long-acting reversible contraception uptake and associated factors among women of reproductive age in Rural Kenya. Their finding showed that 59.8\% of Kenyan women were using injectables.

The finding of the present study illustrated that a minority $15.63 \%$ of the study subjects were implants users. This finding is in harmony with EDHS 2014, as they illustrated that only 1\% of Egyptian women were implants users (El-Zanaty, 2014). This result could be attributed to the fact cited in the literature about implants as most women fear of insertion and removal of implanon, fear of side effects such as weight gain and their beliefs that it may cause cancer and may lost in their body (Banafa, Al-Hanshi, Almualm \& Alkathiri, 2017).

This finding is in line with two Ethiopian studies. First: (Biruk \& Hailemariam, 2015) who conducted a study titled implants contraceptive utilization and factors associated among married women. They revealed that implant contraceptive prevalence rate among married Ethiopian women is only $12 \%$. Second: (Girma, Sultan \& Leges, 2016) who studied prevalence and factors influence utilization of modern contraceptive methods among married women of reproductive age group. They reported that only $15.3 \%$ of Ethiopian women were implants users. In addition to, the previously mentioned one conducted by (Ilori et al., 2017). They reported that only $10 \%$ of Nigerian women used implants . Finally, (Moodley \& Mahomed, 2019) who conducted a study about prevalence and predictors of implanon uptake in Uganda in South Africa. They reported that only $16 \%$ of African women were implants users.

Simultaneously, the same finding is inconsistent with findings of two researches. First: (Mubarik, Jameel \& Khalil, 2016) who conducted a study titled knowledge, attitude and utilization of sub-dermal birth control implants among married women in Pakistan. Second:(Alege, Matovu, Ssensalire \& Nabiwemba, 2016) who conducted a study about knowledge, sources and use of family planning methods among women aged 15-49 years in Uganda. The two studies revealed that as much as $25 \%$ of their participants were implants users.

Overall health beliefs among contraceptive users:

The HBM is a value-expectancy theory. Value-expectancy concepts reformulated in the context of health-related behaviours. The HBM assumes that a course of action (utilization of contraceptives) available to women would be beneficial in reducing either their susceptibility to threat of unplanned pregnancies or problems associated with contraception uptake (Hanson \& Benedict, 2002). However, women's perceptions that anticipated barriers had a role to utilize contraceptives effectively. They weigh the perceived barriers against the perceived benefits. If the perceived barriers outweigh perceived benefits this could contributing to non-utilization of contraceptives (Poss, 2001).

Perception of personal susceptibility and beliefs of seriousness of using contraception are important for influencing the contraceptive behaviour. Generally speaking, in this study more than one- half $(56.56 \%)$ of the study subjects perceived themselves highly susceptible for threat of getting pregnant. This result is a positive finding, since understanding the susceptibility to the threat of unplanned pregnancies will act as a motive toward seeking contraceptives and family planning services which modify the risky behaviours and encourage women to be more adherent with contraceptive method uptake. Specifically, approximately an equal precent of the study subjects (44.69\% $\$ 55.31 \%$ ) of them were either strongly agree or agree that using contraception reduces the chances of unintended pregnancy, respectively. This could be attributed to the fact that study subjects believe that they will become pregnant if they have sex without using any contraceptive method as all the study subjects are within reproductive age and fertile.

This finding is in harmony with five researches. Firstly,(Frost \& Lindberg, 2013)whose study titled reasons for using contraception: Perspectives of US women seeking care at specialized family planning clinics in United States of America. They reported that $63 \%$ among American women agreed that using contraception prevent unplanned pregnancies. Secondly,(Yang, 2013)who conducted a study about health beliefs and contraception use in Haiti .They revealed that most of the Haiti women agree that contraception prevents unplanned pregnancies. Thirdly, (Tabane \& Peu, 2015) who conducted a study titled perceptions of female teenagers in the Tshwane District on the use of contraceptives in South Africa. They reported that most of African females had positive perception toward the use of contraceptives to prevent unplanned pregnancy. Fourthly,(Deri, 2016) who conducted a study titled contraceptive use among women of reproductive age 
in Jirapa District in Ghana. They revealed that $47 \%$ of Ghanaian women agreed that contraceptives prevent unwanted pregnancies. Fifthly,(Adefalu et al., 2018)who had studied awareness and opinions regarding contraception by women of reproductive age in NorthWest Nigeria. They reported that $63.4 \%$ of the Nigerian women agree that contraceptives used to prevent unwanted pregnancy. On the other hand, the same study result in disharmony with (Frohwirth, Moore \& Maniaci, 2013) who studied perceptions of susceptibility to pregnancy among women obtaining abortions in United States of America. Their results showed that American women had low perceived susceptibility as they feel insusceptible to the threat of pregnancy so, didn't use any contraceptive method.

Perceived severity is an important construct in determining compliance to contraceptive behavior. This construct considers personal feelings of the seriousness of contraception use; based upon subjective assessment of medical and social consequences of contraceptives uptake. In other words women's perception about the side-effects of the contraceptive methods and their willingness to tolerate them partly reflects their attractiveness, continuation and adherence to contraceptive methods (Jaccard, Dodge \& Dittus, 2003).

In the current study more than three - fourths $(76.25 \%)$ of the study subjects had moderate perceived severity to the problems associated with contraceptive use. That is to say that only $39.69 \%$ of the study subjects were agreed that using contraception may cause many health problems. This finding could be attributed to the fact that the doctor was the person who made the decision regarding contraceptive utilization and the main source of information to almost one half $(48.44 \%)$ of the study subjects so; the study subjects had correct and reliable information regarding contraceptive methods and its adverse effects. They knew how to manage these problems thus, they experience fewer problems

This result is supported by the findings of two researches. Firstly, (Elkalmi et al., 2015).who conducted a study titled knowledge, awareness, and perception of contraception among senior pharmacy students in Malaysia. They reported that 30\% of Malaysian women agreed that using contraceptive methods may cause health problems. The previously mentioned one done by (Tabane \& Peu, 2015). They illustrated that $40 \%$ of their participants agree that contraceptives can cause many health problems. They believe that contraceptives as COCs cause health problems such as blood clots and weight gain.

On contrary this finding is in disagreement with (Gueye et al., 2015) who had conducted a study titled belief in family planning myths at the individual and community levels and modern contraceptive use in urban Africa. They revealed that $71.5 \%$ of Kenyan women, $46.6 \%$ of Nigerian women and $50.2 \%$ of Senegalese women agreed that using contraceptive methods can cause health problems.(Alharbi, Alharbi, Alnazzawi, Albasri \& Towairqi Mal, 2017) who had conducted a study about knowledge, attitudes and practices towards family planning among Saudi female teachers in Saudi Arabia. They reported that $55.7 \%$ of Saudi women agreed that using contraceptive methods can cause health problems. At last the previously mentioned one (Adefalu et al., 2018). They reported that $69.2 \%$ of the Nigerian women agreed that using contraceptives can cause health problems.

According to the HBM, if no benefits regarding contraceptive uptake are perceived by women, no behavioural change can take place. Several studies illustrated that women are more likely to comply with health recommendations when they believe that these actions will be effective in preventing, detecting, or treating the disease and thus reducing its threat to them (Baiden \& Rajulton, 2011; Jones, Tapales, Lindberg \& Frost, 2015).

In the present study the vast majority $(84.37 \%)$ of the study subjects had high perceived benefits of contraception uptake. Specifically, about three-fourths (74.06\%) of the study subjects strongly agreed that using contraceptives organize family size. As much as (39.38\%) of the study subjects agreed that using contraceptives regulate menstrual cycle and one- half $(51.25 \%)$ of them were agreed that using contraceptives prevent many health problems.

This could be attributed to the awareness of the study subjects about the benefits of contraception uptake. As the majority of the study subjects were educated and only $10.62 \%$ of them were illiterate so, they continue, adhere and comply with contraceptive methods and this is clear as in this study $65 \%$ of the study subjects use the method more than 2 years.

This finding is congruent with the previously mentioned one conducted by(Frost \& Lindberg, 2013).They reported that $63 \%$ of American women strongly agreed that using contraceptives organize family size. Also, (Mohsen et al., 2016) who conducted a study titled effect of application of a health belief model on changing mothers' beliefs regarding birth spacing in Egypt. They reported that all the study subjects strongly agreed that family planning helps decide desirable family size, $42.7 \%$ of them agreed that using contraceptives regulate menstrual cycle and $54.7 \%$ of them agreed that using contraceptives prevent many health problems and improve maternal health.

This finding is incongruent with two researches First:(Okanlawon, Reeves \& Agbaje, 2010) whose study titled contraceptive use: knowledge perceptions and attitudes of refugee youths in Nigeria. They reported that the only $54.3 \%$ of Nigerian women strongly agreed that contraception were good for organizing family. Second: the previously mentioned study with (Elkalmi et al., 2015). They reported that only $40 \%$ of Malaysian women agreed that family planning organize family size.

Perceived barriers are possible blocks or hindrances to engage in contraceptive behaviours, including perceived side effects of contraceptive methods (i.e. weight gain or mood swings), physiological risks of hormonal contraceptives (i.e. blood clots), inconvenience (i.e. having to remember to take a daily pill), and limited access to methods, cost, inconvenience and un availability, fear of infertility and distance to the clinics and long waiting time. According to HBM the subtraction of perceived barriers to preventive actions from perceived benefits of actions is a source that potentially increases the probability of doing preventive acts "contraceptive behaviour"(Etenikang, Uji, Obinna \& Ife, 2017; Palamuleni, 2013).

In the present study Almost all of the subjects had moderate perception to the barriers associated with contraception utilization. That's to say that only $(22.5 \%)$ of the study subjects agreed that using contraceptives can cause infertility, only $18.44 \%$ of the study subjects strongly agreed that using contraceptives may cause menstrual irregularities, and $22.19 \%$ of them agreed that using contraceptives are negatively affected by long distance and waiting time. This finding could be attributed to the efforts done by the Egyptian government to increase the accessibility and availability of different contraceptive methods and increase outlets of family planning services to decrease obstacles that can face the women which is an indicator for increase contraceptive prevalence.

These results are in accordance with the finding of the previously mentioned one conducted by (Elkalmi et al., 2015). They illustrated that only $24.8 \%$ of Malaysian women agreed that using contraceptive increase the risks of infertility. According to (Ouma et al., 2015) who conducted a study titled obstacles to family planning use among rural women in Amuru District, Northern Uganda. They revealed that $32.3 \%$ of women stated that family planning services were far away from home that make them usually came late for the refill and also, they had to wait for a long time for the services. The previously mentioned one conducted by (Asadisarvestani, Khoo, Malek, Yasin \& Ahmadi, 2017). They illustrated that $20.6 \%$ of the urban women agreed that the use of contraceptives can cause infertility. Another study titled knowledge, practice and perception of contraception by literate adolescents in Calabar, Nigeria conducted by (Etenikang et al., 2017). They reported that a minority $14.5 \%$ of Nigerian women agreed that contraception utilization can result in infertility and only $18.1 \%$ of them agreed that contraceptives cause menstrual irregularities.

This result is in disagreement with the previously mentioned study conducted by (Yang, 2013). He reported that $40 \%$ the participants disagreed that using contraceptives are negatively affected by long distance and waiting time. Also, the previously mentioned one 
conducted by (Biruk \& Hailemariam, 2015). They reported that 53\% of Indian women agreed that contraceptive use leads to infertility. Similarly, (Sunnu \& Adatara, 2016) who conducted a study about knowledge, attitudes, and beliefs toward contraceptive use among women and men in Ghana. They revealed that $39.2 \%$ of Ghanaian women agreed that contraceptives use can make them infertile. As well as, the previously mentioned one conducted by (Mohsen et al., 2016). They reported that a minority of Egyptian women agreed that using contraceptives are negatively affected by far distance. Finally, the previously mentioned one conducted by (Asadisarvestani et al., 2017). They illustrated that $43.7 \%$ rural women agreed that the use of contraceptives can cause infertility.

The Health Belief Model is a framework from behavioural science helps define a set of factors specifically suitable to local health beliefs and behaviours so, it is used in order to understand women's health seeking behaviour from an individual level as well as the mechanisms of their decision-making process for contraceptive use. However, yet it rarely used in the contraception field (Yang, 2013).

According to women's health beliefs and their utilization of contraceptive methods in the present study. There was a statistically significant correlation between study subjects' health beliefs and their utilization of contraceptive methods. Namely, perceived susceptibility $(\mathrm{MCP}=$ $0.00)$, perceived severity $(\mathrm{MCP}=0.00)$, perceived benefits $(\mathrm{MCP}=0.00)$ and perceived barriers $(\mathrm{MCP}=0.02)$. Specifically, that there was a statistically significant correlation between the study subjects' perceived susceptibility and their utilization of contraceptive methods. Where substantial proportion $(66.39 \% \& 86.00 \%)$ of IUDs and implants users perceived themselves highly susceptible for threat of getting pregnant compared to only $43.75 \% \& 33.80 \%$ of OCPs and injectables users, respectively. This finding could be attributed to rumours and misconceptions of both IUDs and implants among Egyptian women. Such as IUD can rust or melt on the body and become less effective in preventing pregnancy or it can penetrate or expelled or will not fit her uterus. In addition to their fear that implant could be absorbed or lost in their bodies (Russo, Miller \& Gold, 2013; Yen, Saah \& Hillard, 2010).

Regarding perceived severity, there was a statistically significant correlation between study subjects' perceived severity and their utilization of contraceptive methods. Specifically, two- fifths (40\%) of the study subjects who were implant users had high perceived severity to problem associated with its utilization compared to approximately an equal percent $(25.21 \% \& 27.50 \%)$ of those who were either IUDs or OCPs users, respectively. This could be attributed to that implant users actually experience a lot of side effects such as weight gain and bleeding.

Concerning perceived benefits there was a statistically significant positive correlation between study subjects' perceived benefits $(\mathrm{MCP}=$ $0.00)$, perceived barriers ( $\mathrm{MCP}=0.02)$ and their contraceptive methods utilization. These results reflect an increase in the awareness and perception of the women regarding the benefits of using the contraceptive methods despite presence of some barriers.

These finding is in agreement with four studies. Firstly:(Wiebe, Trouton \& Dicus, 2010). They reported that that despite the discomforts and side effects associated with IUDs. Almost all the Canadian women used IUDs because of its high contraceptive efficacy, long-term convenience and safety. Secondly: (Peipert et al., 2011) who conducted a study title continuation and satisfaction of reversible contraception in the United States of America. Thirdly:(Yoost, 2014) whose study titled understanding benefits and addressing misperceptions and barriers to intrauterine device access among populations in the United States patient. Both studies revealed that most women prefer IUD than other birth control method due to its contraceptive and non- contraceptive benefits. Fourthly:(Roderique-Davies, McKnight, John, Faulkner \& Lancastle, 2016) who conducted a study titled models of health behaviour predict intention to use long acting reversible contraception use in South East Wales in England. They concluded that increasing processes that influence perceived benefits and decreasing the barriers the woman can face will be more effective in increasing contraception uptake.

Modifiable factors:

The core assumption of the health belief model is based on the idea that changing the health belief is the milestone for behaviour change. Modifiable factors play an important role in changing behaviour. As the four major constructs of the health belief model (perceived susceptibility, perceived severity, perceived benefits and perceived barriers) are modified by other factors such as culture, educational level, past experience, skills, economic status and peer pressure. The modifying factors can affect the women decision to use contraceptives.

- Education:

The present study revealed a statistically significant correlation between study subjects' perceived susceptibility $(\mathrm{MCP}=00.00)$, perceived severity $(\mathrm{MCP}=0.040)$ and perceived benefits $(\mathrm{MCP}=00.00)$ and their level of education. That is to say susceptibility to threat of pregnancy increased with increasing level of education. As educated women had better perception and awareness of the threat of unplanned pregnancy and its negative consequences, better understanding that using contraception may associated with some problems and finally they had better perception about benefits of using contraceptive methods.

This result is expected since education usually paves the way to more health information seeking about contraception and had better access to health facilities for utilization of contraceptive methods. Also highly educated mothers are expected to have developed more adequate skills to maintain their adherence with contraceptives method. This finding is self- explainable by the fact that most literature illustrated that educational attainment is believed to have an indirect effect on contraceptive behaviour, through influencing the perception of susceptibility, severity and benefits that overcome to the perceived barriers (Tavafian, 2012).

- Income:

The present study showed a statistically significant correlation between study subjects' perceived susceptibility $(\mathrm{MCP}=00.00)$, perceived barriers $(\mathrm{MCP}=0.040)$ and their income. The susceptibility to threat of pregnancy increases gradually with increasing income unlike the barriers that are decreased with income increase. This could be explained by the fact that socio economic status gave an index of assets that women may have such as cell phones, radios, televisions and cars. All that facilities are important factors to access to information and decrease barriers like transportation problems and far distance of the family planning clinic.

- Occupation:

The present study illustrated a statistically significant correlation between the perceived susceptibility of the subjects $(\mathrm{MCP}=00.00)$, perceived barriers $(\mathrm{MCP}=0.005)$ and their occupation. This finding could be explained as working women may have greater perceived susceptibility concern about threat of pregnancy than non-working women regarding progress in their career women. Also, their work hours may be a barrier in obtaining family planning services and follow up visits. Unfortunately, this result is incomparable because the studies in this area are rare and scare.

\section{Conclusion and recommendations}

\subsection{Conclusion}

Based on the findings of the present study, it can be concluded that: 
Contraceptive users had high perception related to susceptibility of the threat of pregnancy and benefits of contraceptive methods utilization Moreover, the vast majority of them had moderate perception to severity and barriers associated with contraception uptake. Last but not least, there was a statistically significant between women's health beliefs and their utilization of contraceptive method.

\subsection{Recommendations}

Based on the findings of the present study, the following recommendations are suggested:

- Items of health belief scale should be adopted as integral part of family planning assessment sheet.

- Pre- service - and in-service training are recommended for health care providers regarding family planning improvement strategies based on health belief model.

- Health beliefs regarding contraception utilization are suggested to be involved in the maternity curricula.

- Raising public and community awareness about importance of family planning and correction of their misconceptions regarding contraception utilization through mass media and social networks.

\section{References}

[1] Adefalu, A.A., Ladipo, O.A., Akinyemi, O.O., Popoola, O.A., Latunji, O.O., \& Iyanda, O.F. (2018). Awareness and opinions regarding contraception by women of reproductive age in North-West Nigeria. The Pan African Medical Journal, 30. https://doi.org/10.11604/pamj.2018.30.65.12975.

[2] Alemayehu, G.A., Fekadu, A., Yitayal, M., Kebede, Y., Abebe, S.M., Ayele, T.A., . . Gelagay, A.A. (2018). Prevalence and determinants of contraceptive utilization among married women at Dabat Health and Demographic Surveillance System site, northwest Ethiopia. BMC women's health, 18(1), 118. https://doi.org/10.1186/s12905-018-0611-3.

[3] Alege, S.G., Matovu, J.K.B., Ssensalire, S., \& Nabiwemba, E. (2016). Knowledge, sources and use of family planning methods among women aged 15-49 years in Uganda: a cross-sectional study. Pan African Medical Journal, 24(1), 39. https://doi.org/10.11604/pamj.2016.24.39.5836.

[4] Alharbi, M.M., Alharbi, M.S., Alnazzawi, A., Albasri, R., \& Towairqi Mal, S.S. (2017). Knowledge, attitudes and practices towards family planning among Saudi female teachers in Al-Madinah Al-Munawarah City, Saudi Arabia. International journal of pharmaceutical sciences research, 4, 8292.

[5] Ali, A., Ali, S.A., Aziz Ali, S., \& Khuwaja, N.S. (2016). Determinants of Unintended Pregnancy among Women of Reproductive Age in Developing Countries: A Narrative Review. Journal of midwifery and reproductive health, 4(1), 513-521.

[6] Asadisarvestani, K., Khoo, S.L., Malek, N.M., Yasin, S.M., \& Ahmadi, A. (2017). Determinants of contraceptive usage among married women in Shiraz, Iran. Journal of Midwifery and Reproductive Health, 5(4), 1041-1052. https://doi.org/10.17795/whb-38928.

[7] Baiden, P., \& Rajulton, F. (2011). Factors influencing condom use among women in Ghana: an HIV/AIDS perspective. Journal Of Social Aspects Of HIV/AIDS, 8(2), 46-54. https://doi.org/10.1080/17290376.2011.9724985.

[8] Bamufleh, R.A., Al-Zahrani, A.E., \& Yousuf, S.A. (2017). Systematic review: Contraceptive knowledge and use in Saudi Arabia. Journal of Gynaecology and Obstetrics 5, 69-77. https://doi.org/10.11648/j.jgo.20170506.11.

[9] Banafa, N.S., Al-Hanshi, A.S., Almualm, Y., \& Alkathiri, M.O. (2017). Knowledge and Attitude about Side Effect of Implanon (Implant) among Women Attend Primary Health Center-Al Mukalla District Yemen. Acta Scientific Medical Sciences, 1(1), 32-37.

[10] Becker, M.H., Haefner, D.P., Kasl, S.V., Kirscht, J.P., Maiman, L.A., \& Rosenstock, I.M. (1977). Selected psychosocial models and correlates of individual health-related behaviors. Medical care, 15(5), 27-46. https://doi.org/10.1097/00005650-197705001-00005.

[11] Biruk, E., \& Hailemariam, T. (2015). Implants Contraceptive Utilization and Factors Associated among Married Women in the Reproductive Age Group (18-49 Year) in Southern Ethiopia. Journal Women's Health Care, 4(1), 281.

[12] Çalıkoğlu, E.O., Bilge Yerli, E., Kavuncuoğlu, D., Yılmaz, S., Koşan, Z., \& Aras, A. (2018). Use of Family Planning Methods and Influencing Factors Among Women in Erzurum. Medical science monitor : international medical journal of experimental and clinical research, 24, 5027-5034. https://doi.org/10.12659/MSM.908388.

[13] Champion, V.L. (1993). Instrument refinement for breast cancer screening behaviors. Nursing research, 42(3), 139-143. https://doi.org/10.1097/00006199-199305000-00003.

[14] Chernick, L.S., Schnall, R., Higgins, T., Stockwell, M.S., Castano, P.M., Santelli, J., \& Dayan, P.S. (2015). Barriers to and enablers of contraceptive use among adolescent females and their interest in an emergency department based intervention. Contraception, 91(3), 217-225. https://doi.org/10.1016/j.contraception.2014.12.003.

[15] Clifford, E., Johnscy, S., Pratidnya, K., Rachel, J., \& Reshma, R. (2015). A descriptive study to assess the perception and practice of selected contraceptive methods among target population in selected areas of mumbai. Puplished Master thesis, Fortis Institute of Nursing, Mumbai.

[16] De Peralta, A.M. (2011). Health beliefs and socio-cultural factors that predict cervical cancer screening behaviors among Hispanic women in seven cities in the Upstate of South Carolina. PhD Thesis. Clemson Universit.

[17] Deri, M. (2016). Contraceptive use among women of reproductive age in Jirapa District in Ghana. Master thesis, University of Ghana

[18] Eliason, S.K., Bockarie, A.S., \& Eliason, C. (2018). Postpartum fertility behaviours and contraceptive use among women in rural Ghana. Contraception and reproductive medicine, 3(1), 13. https://doi.org/10.1186/s40834-018-0066-9.

[19] Elkalmi, R.M., Khan, M.U., Ahmad, A., Srikanth, A.B., Abdurhaman, N.S., Jamshed, S.Q., . . Ab Hadi, H.B. (2015). Knowledge, awareness, and perception of contraception among senior pharmacy students in Malaysia: A pilot study. Journal of research in pharmacy practice, 4(2), 94. https://doi.org/10.4103/2279-042X.155760.

[20] El Shamy, B.M., Abdelsatar, H.N., \& Awad, M.M. (2013). The contraceptive knowledge and use among women attending primary health care centers of Al Dawahe District in Port Said governorate. The Medical Journal of Cairo University, 81(2), 137-142.

[21] El-Zanaty, F. (2014). Egypt Demographic and Health Survey. Cairo, Egypt: Ministry of Health and Population. El-Zanaty and Associates, National Population Council; 11-2.

[22] Endriyas, M., Eshete, A., Mekonnen, E., Misganaw, T., Shiferaw, M., \& Ayele, S. (2017). Contraceptive utilization and associated factors among women of reproductive age group in Southern Nations Nationalities and Peoples' Region, Ethiopia: cross-sectional survey, mixed-methods. Contraception and reproductive medicine, 2(1), 10. https://doi.org/10.1186/s40834-016-0036-Z.

[23] Envuladu, E., Agbo, H., Abigail., Mohammed, A., Chia, L., Kigbu, J.H., \& Zoakah, A. (2012). Utilization of modern contraceptives among female traders in Jos South LGA of Plateau state, Nigeria. International Journal of Medicine and Biomedical Research, 1(3), 224-231. https://doi.org/10.14194/ijmbr.1310.

[24] Etenikang, A.S., Uji, A.B., Obinna, N.C., \& Ife, E.C. (2017). Knowledge, practice and perception of contraception by literate adolescents in Calabar, Nigeria. European Journal of Biology and Medical Science Research, 5(6), 1-6.

[25] Frohwirth, L., Moore, A.M., \& Maniaci, R. (2013). Perceptions of susceptibility to pregnancy among US women obtaining abortions. Social Science \& Medicine, 99, 18-26 https://doi.org/10.1016/j.socscimed.2013.10.010.

[26] Frost, J.J., \& Lindberg, L.D. (2013). Reasons for using contraception: perspectives of US women seeking care at specialized family planning clinics. Contraception, 87(4), 465-472. https://doi.org/10.1016/j.contraception.2012.08.012.

[27] Gharaibeh, M.K., Oweis, A., Shakhatreh, F.M.N., \& Froelicher, E.S. (2011). Factors Associated with Contraceptive Use among Jordanian Muslim Women: Implications for Health and Social Policy. Journal of International Women's Studies, 12(3), 168-184. 
[28] Girma, T., Sultan, A., \& Leges, K. (2016). Prevalence and Factors Influences Utilization of Modern Contraceptive Methods among Married Women of Reproductive Age Group (15-49 Years) in Holeta Town, Oromia, Ethiopia 2016. Journal of Pregnancy and Child Health, 3(272), 2. https://doi.org/10.4172/2376-127X.1000272.

[29] Gueye, A., Speizer, I.S., Corroon, M., \& Okigbo, C.C. (2015). Belief in Family Planning Myths at the Individual and Community Levels and Modern Contraceptive Use in Urban Africa. International perspectives on sexual and reproductive health, 41(4), 191-199. https://doi.org/10.1363/intsexrephea.41.4.0191.

[30] Hamdy, A. (2014). Pattern of contraception utilization among women attending to the well-baby clinics in damanhour city. Unpuplished Master Thesis, Faculty of Nursing. Alexandria University.

[31] Hettiarachchi, J., \& Gunawardena, N.S. (2012). Factors related to choose of modern vs traditional contraceptives among women in rural Sri Lanka. Sri Lanka Journal of Obstetrics and Gynaecology, 33(1), 1-20. https://doi.org/10.4038/sljog.v33i1.3999.

[32] Huda, F.A., Robertson, Y., Chowdhuri, S., Sarker, B.K., Reichenbach, L., \& Somrongthong, R. (2017). Contraceptive practices among married women of reproductive age in Bangladesh: A review of the evidence. Reproductive health, 14(1), 69. https://doi.org/10.1186/s12978-017-0333-2.

[33] Ilori, O.R., Ilori, O.S., \& Awodutire, P.O. (2017). Contraceptive Practices among Married Women of Reproductive Age Group in Ogbomoso Metropolis, Nigeria. Asian Journal of Medicine and Health, 9(4), 1-9. https://doi.org/10.9734/AJMAH/2017/37743.

[34] Jaccard, J., Dodge, T., \& Dittus, P. (2003). Do adolescents want to avoid pregnancy? Attitudes toward pregnancy as predictors of pregnancy. Journal of Adolescent Health, 33(2), 79-83. https://doi.org/10.1016/S1054-139X(03)00134-4.

[35] Jalang'o, R., Thuita, F., Barasa, S.O., \& Njoroge, P. (2017). Determinants of contraceptive use among postpartum women in a county hospital in rural KENYA. BMC public health, 17(1), 604-606. https://doi.org/10.1186/s12889-017-4510-6.

[36] Jones, R.K., Tapales, A., Lindberg, L.D., \& Frost, J. (2015). Using longitudinal data to understand changes in consistent contraceptive use. Perspectives on sexual and reproductive health, 47(3), 131-139. https://doi.org/10.1363/47e4615.

[37] Kahraman, K., Göç, G., Taşkın, S., Haznedar, P., Karagözlü, S., Kale, B., . . Ozmen, B. (2012). Factors influencing the contraceptive method choice: a university hospital experience. Journal of the Turkish German Gynecological Association, 13(2), 102-105. https://doi.org/10.5152/jtgga.2012.07.

[38] Kassa, N., Berhane, Y., \& Worku, A. (2012). Predictors of unintended pregnancy in Kersa, eastern Ethiopia, 2010. Reproductive health, 9, 1. https://doi.org/10.1186/1742-4755-9-1.

[39] Megabiaw, B. (2012). Awareness and utilization of modern contraceptives among street women in North-West Ethiopia. BMC women's health, 12(1), 31. https://doi.org/10.1186/1472-6874-12-31.

[40] Moodley, A., \& Mahomed, O. (2019). Prevalence and predictors of Implanon uptake in Ugu (Ugu North Sub District) 2016/17. South African Family Practice, 61(2), 48-52. https://doi.org/10.1080/20786190.2018.1548725.

[41] Mohsen, M.M., El-Abbassy, A.A., \& Khalifa, A.M. (2016). Effect of application of a Health Belief Model on changing mothers' beliefs regarding birth spacing in rural areas. Clinical Nursing Studies, 4(3), 54-66. https://doi.org/10.5430/cns.v4n3p54.

[42] Mubarik, M., Jameel, N., \& Khalil, R. (2016). Knowledge, attitude and utilisation of sub-dermal birth control implants among married rural women of Pakistan. International journal of research in medical sciences, 4, 2229-2239. https://doi.org/10.18203/2320-6012.ijrms20161792.

[43] Nyarko, S.H. (2019). Unintended Pregnancy among Pregnant Women in Ghana: Prevalence and Predictors. Journal of Pregnancy and Child Health, 2019, 8. https://doi.org/10.1155/2019/2920491.

[44] Okanlawon, K., Reeves, M., \& Agbaje, O.F. (2010). Contraceptive use: Knowledge, perceptions and attitudes of refugee youths in Oru Refugee Camp, Nigeria. African journal of reproductive health, 14(4), 17-26.

[45] Onokerhoraye, A.G., \& Dudu, J.E. (2016). Onokerhoraye, Johnson Egbemudia Dudu.Knowledge and Practice of Family Planning by Women of Childbearing Age in Delta State, Nigeria. International Journal of Humanities and Social Science Invention, 8(5), 66-75.

[46] Ontiri, S., Ndirangu, G., Kabue, M., Biesma, R., Stekelenburg, J., \& Ouma, C. (2019). Long-Acting Reversible Contraception Uptake and Associated Factors among Women of Reproductive Age in Rural Kenya. International journal of environmental research and public health, 16(9), 1543. https://doi.org/10.3390/ijerph16091543.

[47] Ouma, S., Turyasima, M., Acca, H., Nabbale, F., Obita, K.O., Rama, M., . . Odongo-Aginya, E.I. (2015). Obstacles to family planning use among rural women in Atiak health center IV, Amuru District, northern Uganda. East African medical journal, 92(8), 394-400.

[48] Palamuleni, M.E. (2013). Socio-economic and demographic factors affecting contraceptive use in Malawi. African journal of reproductive health, 17(3), 91-104.

[49] Peipert, J.F., Zhao, Q., Allsworth, J.E., Petrosky, E., Madden, T., Eisenberg, D., \& Secura, G. (2011). Continuation and satisfaction of reversible contraception. Obstetrics and gynecology, 117(5), 1105-1113. https://doi.org/10.1097/AOG.0b013e31821188ad.

[50] Roderique-Davies, G., McKnight, C., John, B., Faulkner, S., \& Lancastle, D. (2016). Models of health behaviour predict intention to use long-acting reversible contraception. Women's Health, 12(6), 507-512. https://doi.org/10.1177/1745505716678231.

[51] Russo, J.A., Miller, E., \& Gold, M.A. (2013). Myths and misconceptions about long-acting reversible contraception (LARC). Journal of Adolescent Health, 52(4), 14-21. https://doi.org/10.1016/j.jadohealth.2013.02.003.

[52] Samim, T. (2014). Prevalence of Family Planning Method Among Married Women Aged 15-49 Years in One Rural Area NadershaKot District of Khost Province of Afghanistan. Master of Science Thesis, A Turkey Hacettepe University, Nkara

[53] Sanders, J.N., Turok, D.K., Gawron, L.M., Law, A., Wen, L., \& Lynen, R. (2017). Two-year continuation of intrauterine devices and contraceptive implants in a mixed-payer setting: a retrospective review. American journal of obstetrics and gynecology, 216(6), 590. https://doi.org/10.1016/j.ajog.2017.02.003.

[54] Sensoy, N., Korkut, Y., Akturan, S., Yilmaz, M., Tuz, C., \& Tuncel, B. (2018). Factors Affecting the Attitudes of Women toward Family Planning. Family Planning, 6(1), 33. https://doi.org/10.5772/intechopen.73255.

[55] Sunnu, E., \& Adatara, P. (2016). Knowledge, Attitudes, and Beliefs toward Contraceptive Use among Women and Men in the Ho Municipality in the Volta Region, Ghana. The Journal of Middle East and North Africa Sciences, 2(9), 305. https://doi.org/10.12816/0032690.

[56] Tabane, N.S., \& Peu, M.D. (2015). Perceptions of female teenagers in the Tshwane District on the use of contraceptives in South Africa. curationis, 38(2), 1-7. https://doi.org/10.4102/curationis.v38i2.1528.

[57] Tavafian, S.S. (2012). Predictors of cervical cancer screening: An application of health belief model. Topics on cervical cancer with an advocacy for prevention, 1-24.

[58] Tessema, G.A., Streak Gomersall, J., Mahmood, M.A., \& Laurence, C.O. (2016). Factors Determining Quality of Care in Family Planning Services in Africa: A Systematic Review of Mixed Evidence. PloS one, 11(11), e0165627. https://doi.org/10.1371/journal.pone.0165627.

[59] Ukegbu, A.U., Onyeonoro, U.U., Nwokeukwu, H.I., \& Okafor, G.O.C. (2018). Contraceptive Method Preferences, Use and Satisfaction among Women of Reproductive Age (15-49 Years) in Umuahia, Abia State, Nigeria. Journal of contraceptive studies, $3(3), 16$.

[60] United Nations. (2015). United Nations, Department of Economic and Social Affairs, Population Division. Trends in Contraceptive Use Worldwide. Available at: https://www.un.org/en/development/desa/population/publications/pdf/family/trendsContraceptiveUse2015Report.pdf. [Accessed in: Mar, 2019].

[61] Wiebe, E.R., Trouton, K.J., \& Dicus, J. (2010). Motivation and experience of nulliparous women using intrauterine contraceptive devices. Journal of Obstetrics and Gynaecology Canada, 32(4), 335-338. https://doi.org/10.1016/S1701-2163(16)34477-2.

[62] World Health Organization (WHO). (2013). Family planning fact sheet. Available at: https://www.who.int/news-room/fact-sheets/detail/family-planning-contraception. [Accessed in: Mar, 2019].

[63] Yang, F. (2013). Health Beliefs and Contraception Use in Leogane, Haiti: A Qualitative Study. Master Thesis. Duke Global Health Institute in the Graduate School of Duke University.

[64] Yanikkerem, E., Ay, S., \& Piro, N. (2013). Planned and unplanned pregnancy: effects on health practice and depression during pregnancy. The journal of obstetrics and gynaecology research, 39(1), 180-187. https://doi.org/10.1111/j.1447-0756.2012.01958.x. 
[65] Yen, S., Saah, T., \& Hillard, P.J.A. (2010). IUDs and adolescents-an under-utilized opportunity for pregnancy prevention. Journal of pediatric and adolescent gynecology, 23(3), 123-128. https://doi.org/10.1016/j.jpag.2009.09.004.

[66] Yoost, J. (2014). Understanding benefits and addressing misperceptions and barriers to intrauterine device access among populations in the United States. Patient preference and adherence, 8(1), 947-957. https://doi.org/10.2147/PPA.S45710.

[67] Zhao, J., Song, F., Ren, S., Wang, Y., Wang, L., Liu, W., . . . Sun, Y. (2012). Predictors of condom use behaviors based on the Health Belief Model (HBM) among female sex workers: a cross-sectional study in Hubei Province, China. PloS one, 7(11), 49542. https://doi.org/10.1371/journal.pone.0049542. 